\title{
A Modular, Chip-based sample delivery system for serial crystallography
}

Aaron Finke $^{1}$, Jennifer Wierman ${ }^{1}$, Olivier Paré-Labrosse ${ }^{2}$, Jessica Besaw ${ }^{2}$, Antoine Sarrachini², Saeed Oghbaey², Michael Cook ${ }^{1}$, Dwayne Miller ${ }^{3}$, Sol Gruner ${ }^{1}$, Marian Szebenyi ${ }^{1}$

${ }^{1}$ MacCHESS, Cornell University, Ithaca, United States, ${ }^{2}$ Department of Chemistry, University of Toronto, Toronto, Canada, ${ }^{3}$ Max Planck Institute for the Structure and Dynamics of Matter, Hamburg, Germany

E-mail: af554@cornell.edu

Collecting complete protein crystal datasets from the combination of partial datasets of several crystal entities, termed serial crystallography (SX), is quickly gaining popularity for difficult biomolecules that are not amenable to standard single-crystal diffraction methods. In the SX experiment, a few frames of diffraction data from several crystalline entities, typically of micron size, are collected and merged together. Because of the small size of the crystals and their typically weak diffraction, $X$-rays of high intensity and small beam size are necessary, such as from X-ray free electron lasers (XFELs) or synchrotron light sources. Application of SX techniques using radiation from synchrotron light sources poses some interesting challenges due to the lower flux compared to XFELs. While the availability of synchrotron facilities is far higher than that of XFELs, the lower flux means that data collected will be weaker. Maximizing data quality while making the most of the inherently weak data collected is paramount.

Our goal is to make SX as routine as single crystal, rotation method protein crystallography is at most synchrotron facilities around the world. To that end, we have worked on optimizing both sample delivery and data collection at the beamline. We have developed a chip-based sample delivery system for use in SX, suitable for data collection at both synchrotrons and FELs.[1] The porous microfabricated chips efficiently remove the solvent from the substrates, reducing background scatter and improving signal-to-noise. In addition, the micropatterned grids on the chip enable automated rastering for efficient data collection. At Beamline G3 of CHESS, we have developed a modular SX setup featuring a stable, intense beam, using in-house developed capillary optics for focusing. We demonstrate how our work on both fronts enables optimal data collection for SX at synchrotron light sources.

[1] Sherrell, D. A.; Foster, A. J.; Hudson, L.; Nutter, B.; O'Hea, J.; Nelson, S.; Pare'-Labrosse, O.; Oghbaey, S.; Miller, R. J. D.; Owen, R. L. (2015) J. Synchrotron Rad. 22, 1372-1378.

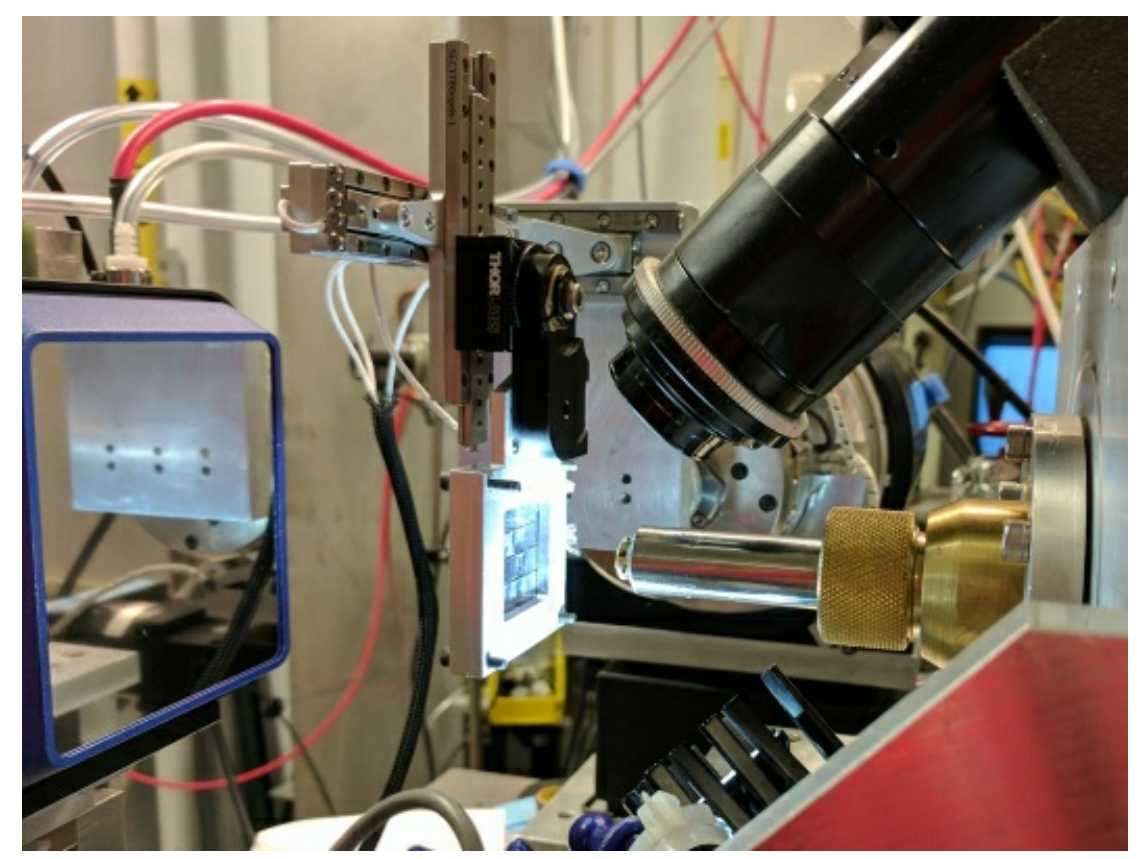

Keywords: serial crystallography, sample delivery, microcrystallography 